Research Article

\title{
The Pricing and Hedging of an Attainable Claim in a Hybrid Black-Scholes Model under Regime Switching
}

\author{
Kuanhou Tian $\mathbb{D},{ }^{1}$ Yanfang $\mathrm{Li}\left(\mathbb{D},{ }^{2}\right.$ and Guixin $\mathrm{Hu} \mathbb{D}^{2}$ \\ ${ }^{1}$ School of Mathematics and Quantitative Economics, Shandong University of Finance and Economics, Jinan, Shandong, China \\ ${ }^{2}$ School of Mathematics and Information Science, Henan Polytechnic University, Jiaozuo, Henan, China \\ Correspondence should be addressed to Yanfang Li; 81746851@qq.com
}

Received 23 January 2021; Accepted 15 November 2021; Published 30 November 2021

Academic Editor: Wei Li

Copyright ( $\odot 2021$ Kuanhou Tian et al. This is an open access article distributed under the Creative Commons Attribution License, which permits unrestricted use, distribution, and reproduction in any medium, provided the original work is properly cited.

This article formulates and dissects a Black-Scholes model with regime switching that can be used to describe the performance of a complete market. An explicit integrand formula $\phi(t, \omega)$ is obtained when the $T$-claim $F(\omega)$ is given for an attainable claim in this complete market. In addition, some perfect results are presented on how to hedge an attainable claim for this Black-Scholes model, and the price $p$ of the European call and the self-financing portfolio $\theta(t)=\left(\theta_{0}(t), \theta_{1}(t)\right)$ are given explicitly. Finally, some concluding remarks are provided to illustrate the theoretical results.

\section{Introduction}

The Black-Scholes model (1973), one of the most important models in modern financial theory, is often used to determine the fair prices of various options. Based on the research involving the classical Black-Scholes model, certain empirical phenomena have received considerable attention recently. The classical Black-Scholes model is often described by the following equations:

$$
\begin{aligned}
X_{0}(t) & =e^{\rho t} \\
\mathrm{~d} X_{i}(t) & =\alpha_{i} X_{i}(t) \mathrm{d} t+\sum_{j=1}^{m} \beta_{i j} X_{i}(t) \mathrm{d} B_{j}(t), \quad i=1,2, \ldots, n,
\end{aligned}
$$

where $\beta_{i j}$ is a $n \times m$ matrix and $B_{j}(t)$ is a Brownian motion. The asset numbers $1,2, \ldots, n$ are risky because of the presence of their diffusion terms and can be used to represent the stock investments. The asset number 0 is risk free due to the absence of the diffusion term, and it can be used to represent a bank investment.

A very natural question is: if the values of $\alpha_{i}$ and $\beta_{i j}$ are random, what will happen to the results? By taking advantage of the ergodic theory of irreducible Markov chain, this paper will provide a perfect result for the case of random $\alpha_{i}$ and $\beta_{i j}$ according to the switching of Markov chain. As an application of our theoretical results, we will answer this question in Example 1.

It is well known that the adjustments of the interest rates by the central banks can produce large disturbances among various options and asset investments. For this reason, it is necessary to consider a switching noise in the Black-Scholes model. In this paper, we adopt the Markov chain to describe this switching noise as in [1-4]. This type of noise can be regarded as a significant fluctuation in the models and can be illustrated as a switching between $n$ regimes.

We are motivated by the work of [5-7] for the option pricing, and we aim to hedge an attainable claim in a normalized market that is described by a stochastic Black-Scholes model with regime switching between two underlying assets that consist of a bond $X_{0}(t)$ and a risky asset $X_{1}(t)$. Under the switching noise (Markov chain) and the white noise (Brownian motions), we give an explicit integrand formula $\phi(t, \omega)$, the price $p$ of the European call, and the self-financing portfolio $\theta(t)=\left(\theta_{0}(t), \theta_{1}(t)\right)$.

\section{Black-Scholes Model}

Suppose that a market is described by $\left(X_{0}(t), X_{1}(t)\right)$, where $X_{0}(t)$ is defined as 


$$
X_{0}(t)=e^{\rho t}
$$

and $X_{1}(t)$ is an Itô process with the form

$$
\mathrm{d} X_{1}(t)=\alpha X_{1}(t) \mathrm{d} t+\sigma \mathrm{d} B(t), \quad X_{1}(0)=x_{1} .
$$

In general, we regularly seek a portfolio $\theta(t)$ to hedge the claim $F(\omega)=\exp \left(X_{1}(T)\right)$ if $\rho, \alpha$, and $\sigma$ are constants (see [8] for more details). A $T$-claim $F$ is usually given by

$$
X_{0}^{-1}(T) F(\omega)=z+\int_{0}^{T} \phi(t, \omega) \mathrm{d} \widetilde{B}(t),
$$

where $X_{0}^{-1}(\cdot)$ and $\phi(t, \omega) \in R^{m}$ satisfy

$$
X_{0}^{-1}(t, \omega)\left(\theta_{0}(t), \ldots, \theta_{n}(t)\right) \sigma(t, \omega)=\phi(t, \omega)
$$

$\theta_{0}(t)$ can also be chosen by the corresponding formula. The portfolio $\theta(t)=\left(\theta_{0}(t), \ldots, \theta_{n}(t)\right)$ is needed to hedge a given claim. It is interesting to find an explicit formula of integrand $\phi(t, \omega)$ for a given $T$-claim $F(\omega)$ to make the portfolio self-financing. Using a generalized version of the Clark-Ocone theorem of the Malliavin calculus, one can find the explicit expression of $\phi(t, \omega)$. To do so, we refer the reader to [9]. There is a simpler method; however, for the Markovian case, see $[8,10]$ for instance. However, there are no results for the regime-switching model, so the aim of this paper is to dissect a more practical model for the integrand formula $\phi(t, \omega)$.

The Black-Scholes model assumes that a market consists of at least one risky asset and one riskless asset. Without loss of generality, here we let a market that has only two securities $X_{0}(t)$ and $X_{1}(t)$, where $X_{0}(t)$ and $X_{1}(t)$ are two Itô processes of the form

$$
\begin{aligned}
& \mathrm{d} X_{0}(t)=\rho X_{0}(t) \mathrm{d} t \\
& \mathrm{~d} X_{1}(t)=\alpha X_{1}(t) \mathrm{d} t+\beta X_{1}(t) \mathrm{d} B(t) .
\end{aligned}
$$

In the literature [8], the authors give the explicit formula for the self-financing portfolio $\theta(t)=\left(\theta_{0}(t), \theta_{1}(t)\right)$ that replicates the $T$-claim $F(\omega)=f\left(X_{1}(T, \omega)\right)$ explicitly. They also mentioned a model as

$$
\begin{aligned}
& \mathrm{d} X_{0}(t)=\rho(t, \omega) X_{0}(t) \mathrm{d} t \\
& \mathrm{~d} X_{1}(t)=\alpha(t, \omega) X_{1}(t) \mathrm{d} t+\beta(t, \omega) X_{1}(t) \mathrm{d} B(t),
\end{aligned}
$$

where $B(t)$ is a 1-dimensional Brownian motion and $\rho(t, \omega), \alpha(t, \omega)$, and $\beta(t, \omega)$ are stochastic processes. (7) belongs to a small class of effectively solvable stochastic differential equations. It is easy to find the solution to equation (7),

$$
\begin{aligned}
& X_{0}(t)=X_{0}(0) \exp \left\{\int_{0}^{t} \rho(s, \omega) \mathrm{d} s\right\}, \\
& X_{1}(t)=X_{1}(0) \exp \left\{\int_{0}^{t} \beta(s, \omega) \mathrm{d} B(s)+\int_{0}^{t}\left(\alpha(s, \omega)-\frac{1}{2} \beta^{2}(s, \omega)\right) \mathrm{d} s\right\},
\end{aligned}
$$

explicitly, if and only if

$$
E\left[\exp \left(\frac{1}{2} \int_{0}^{T} \frac{(\alpha(s, \omega)-\rho(s, \omega))^{2}}{\beta^{2}(s, \omega)} \mathrm{d} s\right)\right]<\infty
$$

Suppose that there exists an equivalent martingale measure $Q$ given by

$$
\mathrm{d} Q(\omega)=\exp \left(-\int_{0}^{T} u(t, \omega) \mathrm{d} B(t)-\frac{1}{2} \int_{0}^{T} u^{2}(t, \omega) \mathrm{d} t\right) \mathrm{d} P(\omega)
$$

Under this martingale measure $Q$, by the Girsanov theorem II, the process

$$
\widetilde{B}(t):=\int_{0}^{t} u(s, \omega) \mathrm{d} s+B(t) .
$$

is a Q-Brownian motion. Thus, equation (7) can be rewritten as

$$
\begin{aligned}
& \mathrm{d} X_{0}(t)=\rho(t, \omega) X_{0}(t) \mathrm{d} t, \\
& \mathrm{~d} X_{1}(t)=\rho(t, \omega) X_{1}(t) \mathrm{d} t+\beta(t, \omega) X_{1}(t) \mathrm{d} \widetilde{B}(t),
\end{aligned}
$$

in terms of this Q-Brownian motion $\widetilde{B}(t)$. Suppose that the market defined by equation (12) has no arbitrage and it is complete. Moreover, only this information for the European option defined by equation (7) is known. Note that the coefficients $\alpha(t, \omega)$ and $\beta(t, \omega)$ in equation (7) are dependent on the random variable $\omega \in \Omega$ in an unknown way. The portfolio $\theta(t)$ for the $T$-claim $F(\omega)$ and the price $p=p(F)$ at $t=0$ of the European options with $T$-claim $F(\omega)$ cannot be defined explicitly. But, when $\rho(t, \omega)=\rho(t)$ and $\beta(t, \omega)=$ $\beta(t)$ are deterministic, the authors in [8] give the price at $t=0$ of a European option with payoff given by a contingent $T$-claim

$$
F(\omega)=f\left(X_{1}(T, \omega)\right)
$$

for some lower bounded function $f: R \longrightarrow R$ such that

$$
E_{Q}\left[f\left(X_{1}(T)\right)\right]<\infty .
$$

The price $p=p(F)$ at time $t=0$ of a European option with payoff given by a contingent $T$-claim in equation (13) has the explicit form as 


$$
p=\xi(T) E_{Q}\left[f\left(x_{1} \exp \left(\int_{0}^{T} \beta(s) \mathrm{d} \widetilde{B}(s)+\int_{0}^{T}\left(\rho(s)-\frac{1}{2} \beta^{2}(s)\right) \mathrm{d} s\right)\right)\right]
$$

where $\xi(t)=X_{0}^{-1}(t)$ and $\widetilde{B}(t)$ is a Q-Brownian motion.

Moreover, if $\rho, \alpha$, and $\beta$ are constants and $\beta \neq 0$, it is a very important special case of equation (7); the price $p$ of a European call option and the self-financing portfolio $\theta(t)=\left(\theta_{0}(t), \theta_{1}(t)\right)$ have been given explicitly in [8]. Considering some practical meanings, we will discuss the case of $\rho, \alpha$, and $\beta$ in equation (7) dependent on $\omega$ in the form of Markov chain. We will give some perfect results by the method of the ergodic theory of an irreducible Markov chain.

In the following part of our paper, we will discuss the Black-Scholes model under regime switching, which is a particular case of equation (7), that is to say, $\rho(t, \omega), \alpha(t, \omega)$, and $\beta(t, \omega)$ are dependent on $\omega$ in the form of Markov chain. At the same time, the model in this paper is an extension of the classical Black-Scholes model and we will give some more perfect results than in [8]. Without loss of generality, we firstly discuss a market $X_{0}(t)$ and $X_{1}(t)$ that is formulated by a Black-Scholes model under regime switching.

\section{Pricing and Hedging of an Attainable Claim}

Throughout the paper, unless otherwise specified, let $\left(\Omega, \mathscr{F}, \mathscr{F}_{t}, P\right)$ be a complete probability space with a filtration $\left\{\mathscr{F}_{t}\right\}_{t \geq 0}$ satisfying the usual conditions (it is rightcontinuous and increasing while $\mathscr{F}_{0}$ contains all P-null sets). Let $B(t)$ be a 1-dimensional standard Brownian motion defined on a complete probability space.

If we consider switching noise (Markov chain) in the classical Black-Scholes model, we can find a Black-Scholes model under regime switching that has the form

$$
\begin{aligned}
& \mathrm{d} X_{0}(t)=\rho(r(t)) X_{0}(t) \mathrm{d} t, \quad X_{0}(0)=1, \\
& \mathrm{~d} X_{1}(t)=\alpha(r(t)) X_{1}(t) \mathrm{d} t+\beta(r(t)) X_{1}(t) \mathrm{d} B(t), \quad X_{1}(0)=x_{1}>0,
\end{aligned}
$$

where $r(t)$ is a right-continuous Markov chain taking values in a finite state space $\mathbb{S}=\{1,2\}$. The generator $\Gamma=\left(\gamma_{i j}\right)_{N \times N}$ of $r(t)$ is given by

$$
\mathbb{P}\{r(t+\delta)=j \mid r(t)=i\}= \begin{cases}\gamma_{i j} \delta+o(\delta), & \text { if } i \neq j, \\ 1+\gamma_{i j} \delta+o(\delta), & \text { if } i=j,\end{cases}
$$

where $\delta>0$ and $\gamma_{i j} \geq 0$ are the transition rate from $i$ to $j$ satisfying $\gamma_{i j}>0$ if $i \neq j$ while $\gamma_{i i}=-\sum_{j \neq i} \gamma_{i j}$.

We also assume that the Markov chain is irreducible which means that Markov chain $r(t)$ has a unique stationary (probability) distribution $\pi=\left(\pi_{1}, \pi_{2}, \ldots, \pi_{N}\right) \in R^{1 \times N}$ that can be determined by solving the following linear equation:

$$
\pi \Gamma=0,
$$

subject to

$$
\begin{aligned}
\sum_{k=1}^{N} \pi_{k} & =1, \\
\pi_{k} & >0, \quad \text { for } \forall k \in \mathbb{S} .
\end{aligned}
$$

Therefore, equation (16) can be regarded as the results of the following equations:

$$
\begin{aligned}
& \mathrm{d} X_{0}(t)=\rho(1) X_{0}(t) \mathrm{d} t, \\
& \mathrm{~d} X_{1}(t)=\alpha(1) X_{1}(t) \mathrm{d} t+\beta(1) X_{1}(t) \mathrm{d} B(t), \\
& \mathrm{d} X_{0}(t)=\rho(2) X_{0}(t) \mathrm{d} t, \\
& \mathrm{~d} X_{1}(t)=\alpha(2) X_{1}(t) \mathrm{d} t+\beta(2) X_{1}(t) \mathrm{d} B(t),
\end{aligned}
$$

switching from one to the other according to the movement of Markov chain $r(t)$. Suppose that the stationary (probability) distribution of Markov chain is $\pi=\left(\pi_{1}, \pi_{2}\right)$ and the initial distribution of $r(t)$ is also $\pi=\left(\pi_{1}, \pi_{2}\right)$. Then, for any $t \geq 0$, the Markov chain $r(t)$ has a stationary distribution $\pi=$ $\left(\pi_{1}, \pi_{2}\right)$ because it is irreducible. Note that the solution of equation (16) is

$$
\begin{aligned}
& X_{0}(t)=\exp \left\{\int_{0}^{t} \rho(r(s)) \mathrm{d} s\right\}, \\
& X_{1}(t)=x_{1} \exp \left\{\int_{0}^{t} \beta(r(s)) \mathrm{d} B(s)+\int_{0}^{t}\left(\alpha(r(s))-\frac{1}{2} \beta^{2}(r(s))\right) \mathrm{d} s\right\} .
\end{aligned}
$$

We all know that a business cycle is often divided into two or more different states, called "expansion" and "contraction" in financial economics. A growing economy is frequently described as being in expansion. For this, we can let $r(t)=$ 
$1, \rho(r(t))=\rho(1), \alpha(r(t))=\alpha(1)$ and $\beta(r(t))=\beta$ (1). We can take the value $r(t)=2, \rho(r(t))=\rho(2), \alpha(r(t))=\alpha(2)$ and $\beta(r(t))=\beta(2)$ to represent the state in contraction. More generally, we can use the state space $\mathbb{S}=\{1,2, \ldots, N\}$ for the value of $r(t)$ to model more complex business cycle structures. In this section, without loss of generality, we consider only two states for a market $\left(X_{0}(t), X_{1}(t)\right)$ by using equation (16).

Theorem 1 (see [8]). Suppose that a market $X(t)$ in terms of $\widetilde{B}(t)$ has the following form:

$$
\begin{aligned}
\mathrm{d} X_{0}(t) & =\rho(X(t)) X_{0}(t) \mathrm{d} t \\
\mathrm{~d} X_{i}(t) & =\rho(X(t)) X_{i}(t) \mathrm{d} t+\sigma_{i}(X(t)) X_{1}(t) \mathrm{d} \widetilde{B}(t), \quad 1 \leq i \leq n .
\end{aligned}
$$

And, assume that $h_{0}: R^{n+1} \longrightarrow R$ is a given function such that

$$
\left\{\frac{\partial}{\partial x_{i}} E_{Q}^{x}\left[\xi(T-t) h_{0}(X(T-t))\right]\right\}_{i=1}^{n},
$$

exists and

$$
E_{Q}^{x}\left[\int_{0}^{T} \phi^{2}(t, \omega) \mathrm{d} t\right]<\infty
$$

where

$$
\phi(t, \omega):=\sum_{i=1}^{n} \frac{\partial}{\partial x_{i}} E_{Q}^{x}\left[\xi(T-t) h_{0}(Y(T-t))\right]_{x=X(t)} \sigma_{i}(X(t)) .
$$

Then, we have the Ito representation formula

$$
\xi(T) h_{0}(X(T))=E_{Q}\left[\xi(T) h_{0}(X(t))\right]+\int_{0}^{T} \phi(t, \omega) \mathrm{d} \widetilde{B}(t) .
$$

Remark 1. Note that the solution of equation (16) is Markovian and this makes it possible to apply the result of Theorem 1 to find $\phi(t, \omega)$ in equation (4).
Lemma 1. Let $r(t)$ be a stationary Markov chain taking value in a finite state space $S=\{1,2, \ldots, N\}$. Then, $Y(t)=$ $\int_{0}^{t} \beta(r(s)) d B(s)$ is a Gaussian process for any $t>0$.

Proof. We assume that the initial distribution of $r(s)$ is $\pi=\left(\pi_{1}, \pi_{2}, \ldots, \pi_{N}\right)$; then,

$$
Y(t)=\int_{0}^{t} \beta(r(s)) \mathrm{d} B(s)=\sum_{i=1}^{N}\left(\pi_{i} \beta(i)\right) B(t) .
$$

Recall that $B(t)$ is a Gaussian process, so it is easy to see that for any $t_{0} \geq 0$, the random variable $Y\left(t_{0}\right)$ is normally distributed with mean 0 and variance $\left[\sum_{i=1}^{N}\left(\pi_{i} \beta(i)\right)\right]^{2} t_{0}$; hence, $Y(t)$ is a Gaussian process.

Remark 2. By Lemma 1, we are able to study the hedging of an attainable claim of a European option defined by a Black-Scholes model with Markovian switching. In the following, we consider a situation where a market has just two securities; we let $X_{0}(t)$ be a risk free asset and $X_{1}(t)$ a risky asset that is an Itô process with the form of equation (16). We have the following result for the hedging of an attainable claim for this situation.

Theorem 2. Suppose that a market is described by $X(t)=$ $\left(X_{0}(t), X_{1}(t)\right)$ which is given by equation (16) with $\rho(r(t)), \alpha(r(t)), \beta(r(t))$ satisfying

$$
E\left[\exp \left(\frac{1}{2} \int_{0}^{T} \frac{(\alpha(r(s))-\rho(r(s)))^{2}}{\beta^{2}(r(s))} \mathrm{d} s\right)\right]<\infty,
$$

for $r(s)$ taking values in $\{1,2\}$. Then, we have the following:

(i) The market $\{X(t)\}$ is no arbitrage and complete, and the price at time $t=0$ of the European T-claim $F(\omega)=f\left(X_{1}(T, \omega)\right)$ is

$$
p=\frac{\xi(T)}{\Delta \sqrt{2 \pi}} \int_{R} f\left(x_{1} \exp \left[y+\int_{0}^{T}\left(\rho(r(s))-\frac{1}{2} \beta^{2}(r(s))\right) \mathrm{d} s\right]\right) \exp \left(-\frac{y^{2}}{2 \Delta^{2}}\right) \mathrm{d} y
$$

where $E_{Q}\left[f\left(X_{1}(T, \omega)\right)\right]<\infty$ and with

$$
\begin{aligned}
\xi(T) & =e^{-\int_{0}^{T} \rho(r(s)) \mathrm{d} s}=e^{-\left(\pi_{1} \rho(1)+\pi_{2} \rho(2)\right) T}, \\
\Delta^{2} & =\int_{0}^{T} \beta^{2}(r(s)) \mathrm{d} s=\left[\pi_{1} \beta^{2}(1)+\pi_{2} \beta^{2}(2)\right] T .
\end{aligned}
$$

(ii) If $f \in C^{1}(R)$, then the self-financing portfolio $\theta(t)=$ $\left(\theta_{0}(t), \theta_{1}(t)\right)$ needed to replicate the T-claim $F(\omega)=$ $f\left(X_{1}(T, \omega)\right)$ is given by

$$
\begin{aligned}
\theta_{1}(t)= & \frac{e_{0}^{T} \rho(r(s)) \mathrm{d} s}{\sqrt{2 \pi(T-t)}} \int_{R} f^{\prime}\left(X _ { 1 } ( t , \omega ) \operatorname { e x p } \left\{\left(\pi_{1} \beta(1)+\pi_{2} \beta(2)\right) x+\int_{0}^{T-t} \rho(r(s))\right.\right. \\
& \left.\left.-\frac{1}{2} \beta^{2}(r(s))\right) \mathrm{d} s\right\} \cdot \exp \left\{\left(\pi_{1} \beta(1)+\pi_{2} \beta(2)\right) x+\int_{0}^{T-t}\left(\rho(r(s))-\frac{1}{2} \beta^{2}(r(s))\right) \mathrm{d} s\right\} \cdot \exp \left\{-\frac{x^{2}}{2(T-t)}\right\} \mathrm{d} x,
\end{aligned}
$$


and $\theta_{0}(t, \omega)$ is determined by

$\theta_{0}(t)=V^{\theta}(0)+\xi(t) A(t)+\int_{0}^{t} \rho(r(s)) \xi(s) \mathrm{d} s$,

where $A(t)=\int_{0}^{t} \theta_{1}(s) d X_{1}(s)+\theta_{1}(t) X_{1}(t) \quad$ and $V^{\theta}(0)=p$.

Proof

(i) It is easy to observe that there exists a process $u(t, \omega)$ which satisfies

$$
\beta(r(t)) X_{1}(t) u(t, \omega)=\alpha(r(t)) X_{1}(t)-\rho(r(t)) X_{1}(t) .
$$

Then, equation (28) implies that

$$
E\left[\exp \left(\frac{1}{2} \int_{0}^{T} u^{2}(s, \omega) \mathrm{d} s\right)\right]<\infty
$$

Define the measure $Q$ on $\mathscr{F}_{T}$ by

$$
\mathrm{d} Q(\omega)=\exp \left(-\int_{0}^{T} u(t, \omega) \mathrm{d} B(t)-\frac{1}{2} \int_{0}^{T} u^{2}(t, \omega) \mathrm{d} t\right) \mathrm{d} P(\omega)
$$

Then, $Q \sim P$ and by the Girsanov theorem II (see $[8,11,12])$, the process

$$
\widetilde{B}(t):=\int_{0}^{t} u(s, \omega) \mathrm{d} s+B(t)
$$

is a Q-Brownian motion. By Theorem 12.1.8 and Theorem 12.2 .5 of [8], the market is complete with no arbitrage opportunity. Therefore, the price at $t=$ 0 of the European option with payoff given by a contingent $T$-claim $F(\omega)=f\left(X_{1}(T, \omega)\right)$ is

$$
p(F)=E_{Q}[\xi(T) F] .
$$

That is,

$$
p=\xi(T) E_{Q}\left[f\left(x_{1} \exp \left(\int_{0}^{T} \beta(r(s)) \mathrm{d} \widetilde{B}(s)+\int_{0}^{T}\left(\rho(r(s))-\frac{1}{2} \beta^{2}(r(s))\right) \mathrm{d} s\right)\right)\right] .
$$

By Lemma 1 , under the measure $Q$, the random variable $Y=\int_{0}^{T} \beta(r(s)) \mathrm{d} \widetilde{B}(s)$ is normally distributed with mean 0 and variance:

$\Delta^{2}=\int_{0}^{T} \beta^{2}(r(s)) \mathrm{d} s=\left[\pi_{1} \beta^{2}(1)+\pi_{2} \beta^{2}(2)\right] T$.

By the definition of the expectation of the function of random variables, $p$ can be expressed explicitly as equation (29). (ii) In terms of $\widetilde{B}(t)$, we rewrite the second equation of equation (16) as

$\mathrm{d} X_{1}(t)=\rho(r(t)) X_{1}(t) \mathrm{d} t+\beta(r(t)) X_{1}(t) \mathrm{d} \widetilde{B}(t)$.

So, we seek the portfolio as

$\theta_{1}(t, \omega)=X_{0}(t)\left(\beta(r(t)) X_{1}(t, \omega)\right)^{-1} \phi(t, \omega)$,

where

$$
\phi(t, \omega):=\frac{\partial}{\partial x} E_{Q}^{x}\left[\xi(T-t) h_{0}\left(X_{1}(T-t)\right)\right]_{x=X_{1}(t)} \beta(r(t)) X_{1}(t),
$$

with $h_{0}(y)=f(y)$ and

$$
\begin{aligned}
X_{1}(t) & =x_{1} \exp \left\{\int_{0}^{t} \beta(r(s)) \mathrm{d} \widetilde{B}(s)+\int_{0}^{t}\left(\rho(r(s))-\frac{1}{2} \beta^{2}(r(s))\right) \mathrm{d} s\right\} \\
& =x_{1} \exp \left\{\left(\pi_{1} \beta(1)+\pi_{2} \beta(2)\right) \widetilde{B}(t)+\left[\pi_{1}\left(\rho(1)-\frac{1}{2} \beta^{2}(1)\right)+\pi_{2}\left(\rho(2)-\frac{1}{2} \beta^{2}(2)\right)\right] t\right\} .
\end{aligned}
$$

Hence, 


$$
\begin{aligned}
& \theta_{1}(t, \omega)=\exp \left\{\int_{0}^{t} \rho(r(s)) \mathrm{d} s\right\} \frac{\partial}{\partial x} E_{Q}^{x_{1}}\left[\exp \left\{\int_{0}^{T-t} \rho(r(s)) \mathrm{d} s\right\} f\left(X_{1}(T-t)\right)\right]_{x=X_{1}(t)} \\
& =\exp \left\{\int_{0}^{t} \rho(r(s)) \mathrm{d} s\right\} \frac{\partial}{\partial x} E_{Q}^{x_{1}}\left[f \left(x _ { 1 } \operatorname { e x p } \left\{\int_{0}^{T-t} \beta(r(s)) \mathrm{d} \widetilde{B}(s)\right.\right.\right. \\
& \left.+\int_{0}^{T-t}\left(\rho(r(s))-\frac{1}{2} \beta^{2}(r(s))\right) \mathrm{d} s\right\}_{x_{1}=X_{1}(t)} \\
& =\exp \left\{\int_{0}^{t} \rho(r(s)) \mathrm{d} s\right\} E_{Q}^{x_{1}}\left[f ^ { \prime } \left(x _ { 1 } \operatorname { e x p } \left\{\int_{0}^{T-t} \beta(r(s)) \mathrm{d} \widetilde{B}(s)\right.\right.\right. \\
& \left.+\int_{0}^{T-t}\left(\rho(r(s))-\frac{1}{2} \beta^{2}(r(s))\right) \mathrm{d} s\right\} \exp \left\{\int_{0}^{T-t} \beta(r(s)) \mathrm{d} \widetilde{B}(s)\right. \\
& \left.+\int_{0}^{T-t}\left(\rho(r(s))-\frac{1}{2} \beta^{2}(r(s))\right) \mathrm{d} s\right\}_{x_{1}=X_{1}(t)} \\
& =\frac{\exp \left\{\int_{0}^{t} \rho(r(s)) \mathrm{d} s\right\}}{\sqrt{2 \pi(T-t)}} \int_{R} f^{\prime}\left(X _ { 1 } ( t , \omega ) \operatorname { e x p } \left\{\left(\pi_{1} \beta(1)+\pi_{2} \beta(2)\right) x\right.\right. \\
& \left.+\int_{0}^{T-t}\left(\rho(r(s))-\frac{1}{2} \beta^{2}(r(s))\right) \mathrm{d} s\right\} \\
& \exp \left\{\left(\pi_{1} \beta(1)+\pi_{2} \beta(2)\right) x+\int_{0}^{T-t}\left(\rho(r(s))-\frac{1}{2} \beta^{2}(r(s))\right) \mathrm{d} s\right\} \exp \left\{-\frac{x^{2}}{2(T-t)}\right\} \mathrm{d} x \text {, }
\end{aligned}
$$

which is assertion equation (31) and this completes the proof.
Considering the irreducible of the Markov chain $r(t)$, we can get

$$
\begin{aligned}
\int_{0}^{T-t}\left(\rho(r(s))-\frac{1}{2} \beta^{2}(r(s))\right) \mathrm{d} s & =\left[\pi_{1}\left(\rho(1)-\frac{1}{2} \beta^{2}(1)\right)+\pi_{2}\left(\rho(2)-\frac{1}{2} \beta^{2}(2)\right)\right](T-t) \\
\int_{0}^{T} \rho(r(s)) \mathrm{d} s & =\left(\pi_{1} \rho(1)+\pi_{2} \rho(2)\right) T .
\end{aligned}
$$

So, for the self-financing portfolio $\theta(t)$ of a market described by a stochastic Black-Scholes model with
Markovian switching, we find a perfect result than assertion equation (31) as

$$
\begin{aligned}
\theta_{1}(t)= & \frac{e^{\left(\pi_{1} \rho(1)+\pi_{2} \rho(2)\right) T}}{\sqrt{2 \pi(T-t)}} \int_{R} f^{\prime}\left(X _ { 1 } ( t , \omega ) \operatorname { e x p } \left\{\left(\pi_{1} \beta(1)+\pi_{2} \beta(2)\right) x\right.\right. \\
& \left.+\left[\pi_{1}\left(\rho(1)-\frac{1}{2} \beta^{2}(1)\right)+\pi_{2}\left(\rho(2)-\frac{1}{2} \beta^{2}(2)\right)\right](T-t)\right\} \\
& \cdot \exp \left\{\left(\pi_{1} \beta(1)+\pi_{2} \beta(2)\right) x+\left[\pi_{1}\left(\rho(1)-\frac{1}{2} \beta^{2}(1)\right)+\pi_{2}\left(\rho(2)-\frac{1}{2} \beta^{2}(2)\right)\right](T-t)\right\} \cdot \exp \left\{-\frac{x^{2}}{2(T-t)}\right\} \mathrm{d} x .
\end{aligned}
$$


Remark 3. When $\rho(1)=\rho(2)=\rho, \alpha(1)=\alpha(1)=\alpha$, and $\beta(1)=\beta(2)=\beta$, Theorem 2 reduces to the classical stochastic Black-Scholes formula. The T-claim $F(\omega)$ is given in Theorem 2 according to the movement of Markov chain. The results indicated that $T$-claim $F(\omega)$ is dependent on the randomness of Markov chain, which extends the classical Black-Scholes model without Markovian switching.
Remark 4. Applications to the pricing and hedging of the European call option: we know that the $T$-claims of the European call option are

$$
F(\omega)=\left(X_{1}(T, \omega)-K\right)^{+}
$$

where the exercise price $K>0$ is a constant. Then, the price $p$ at time 0 is

$$
p=x_{1} \Phi\left(\eta+\frac{1}{2}\left(\pi_{1} \beta(1)+\pi_{2} \beta(2)\right) \sqrt{T}\right)-K e^{-\left(\pi_{1} \rho(1)+\pi_{2} \rho(2)\right) T} \Phi\left(\eta-\frac{1}{2}\left(\pi_{1} \beta(1)+\pi_{2} \beta(2)\right) \sqrt{T}\right)
$$

where

$$
\begin{aligned}
\eta & =\left(\pi_{1} \beta(1)+\pi_{2} \beta(2)\right)^{-1} T^{-(1 / 2)}\left(\ln \frac{x_{1}}{K}+\left(\pi_{1} \rho(1)+\pi_{2} \rho(2)\right) T\right), \\
\Phi(y) & =\frac{1}{\sqrt{2 \pi}} \int_{-\infty}^{y} e^{-(1 / 2) x^{2}} \mathrm{~d} x, \quad y \in R,
\end{aligned}
$$

is the standard normal distribution function. Moreover, the replicating portfolio $\theta(t)=\left(\theta_{0}(t), \theta_{1}(t)\right)$ for the claim $F(\omega)$ in (47) is given by

$$
\begin{aligned}
\theta_{1}(t, \omega)= & \Phi\left(( \pi _ { 1 } \beta ( 1 ) + \pi _ { 2 } \beta ( 2 ) ) ^ { - 1 } ( T - t ) ^ { - 1 / 2 } \left(\ln \frac{X_{1}(t)}{K}+\left(\pi_{1} \rho(1)+\pi_{2} \rho(2)\right)(T-t)\right.\right. \\
& +\frac{1}{2}\left(\pi_{1} \beta(1)+\pi_{2} \beta(2)\right)^{3}(T-t) .
\end{aligned}
$$

$\theta_{1}(t, \omega)>0, t \in[0, T]$ means that we can replicate the European call without short selling. For the European put options with $T$-claims $F(\omega)=\left(K-X_{1}(T, \omega)\right)^{+}$, it should be $\theta_{1}(t, \omega)<0, t \in[0, T]$ which means that we have to short sell to replicate the European put option.

In fact, the price $p$ at time 0 given in equation (48) follows by applying Theorem 2 to the function

$$
f(x)=(x-K)^{+} .
$$

It does not matter that the function $f(x)$ is not $C^{1}$ because an approximation argument shows that equation (31) or (46) still holds if we represent $f^{\prime}$ by

$$
f^{\prime}(x)=\chi_{[K, \infty)}(x)
$$

To illustrate our theoretical results, we provide the following example for the pricing, hedging, and an appropriate portfolio of a given attainable claim.

Example 1. Suppose that a market is $\left(X_{0}(t), X_{1}(t)\right)$, where $X_{0}(t)$ obeys that

$$
\mathrm{d} X_{0}(t)=\rho(r(t)) X_{0}(t) \mathrm{d} t, \quad X_{0}(0)=1,
$$

and $X_{1}(t)$ is an Ornstein-Uhlenbeck process with Markovian switching as

$$
\mathrm{d} X_{1}(t)=\alpha(r(t)) X_{1}(t) \mathrm{d} t+\sigma(r(t)) \mathrm{d} B(t), \quad X_{1}(0)=x_{1}>0,
$$

where $r(t)$ is an irreducible Markov chain taking values in $\mathbb{S}=\{1,2\}$. Let $\sigma(1)=\sigma_{1}, \sigma(2)=\sigma_{1}, \quad \rho(1)=\rho_{1}$, and $\rho(2)$ $=\rho_{2}$. We can seek that the portfolio $\theta(t)=\left(\theta_{0}(t), \theta_{1}(t)\right)$ is

$$
\theta_{1}(t, \omega)=X_{0}(t)\left(\sigma(r(t))^{-1} \phi(t, \omega)\right.
$$

where $\phi(t, \omega)$ and $V(0)=z$ are uniquely given by

$$
\xi(T) F(\omega)=z+\int_{0}^{T} \phi(t, \omega) \mathrm{d} \widetilde{B}(t),
$$

and the attainable claim is

$$
F(\omega)=\exp \left(X_{1}(T)\right)=z e^{\left(\pi_{1} \rho_{1}+\pi_{2} \rho_{2}\right) T}+\int_{0}^{T} \phi_{0}(t, \omega) \mathrm{d} \widetilde{B}(t)
$$


with $\phi_{0}(t, \omega)=e^{\left(\pi_{1} \rho_{1}+\pi_{2} \rho_{2}\right) T} \phi(t, \omega)$. Then, by Theorem 1, we can find $\phi(t, \omega)$ and the portfolio $\theta(t)$ explicitly.

In fact, we can rewrite equation (54) in terms of $\widetilde{B}(t)$ as $\mathrm{d} X_{1}(t)=\rho(r(t)) X_{1}(t) \mathrm{d} t+\sigma(r(t)) \mathrm{d} B(t), \quad X_{1}(0)=x_{1}$.
Then, the solution to equation (58) can be explicitly expressed as

$$
\begin{aligned}
X_{1}(t) & =x_{1} e^{\int_{0}^{t} \rho(r(s)) \mathrm{d} s}+\int_{0}^{t} \sigma(r(s)) e^{\int_{0}^{t} \rho(\xi(\tau)) \mathrm{d} \tau} \mathrm{d} \widetilde{B}(s) \\
& =x_{1} e^{\left(\pi_{1} \rho_{1}+\pi_{2} \rho_{2}\right) t}+\left(\pi_{1} \sigma_{1}+\pi_{2} \sigma_{2}\right) \int_{0}^{t} e^{\left(\pi_{1} \rho_{1}+\pi_{2} \rho_{2}\right)(t-s)} \mathrm{d} \widetilde{B}(s) .
\end{aligned}
$$

From equation (59), we know that the solution to equation (58) obeys the normal distribution for any $t \geq 0$. The mean of $X(t)$ is

$$
E X(t)=E x e^{\left(\pi_{1} \rho_{1}+\pi_{2} \rho_{2}\right) t}=e^{\left(\pi_{1} \rho_{1}+\pi_{2} \rho_{2}\right) t} E x=0,
$$

and the variance is

$$
V(X(t))=E\left(X^{2}(t)\right)-[E X(t)]^{2}=E\left(X^{2}(t)\right)=\frac{\left(\pi_{1} \sigma_{1}+\pi_{2} \sigma_{2}\right)^{2}}{2\left|\pi_{1} \rho_{1}+\pi_{2} \rho_{2}\right|} .
$$

So, $X(t)$ obeys the normal distribution $N\left(0,\left(\left(\pi_{1} \sigma_{1}\right.\right.\right.$ $\left.\left.\left.+\pi_{2} \sigma_{2}\right)^{2} / 2\left|\pi_{1} \rho_{1}+\pi_{2} \alpha_{2}\right|\right)\right)$ if the initial value $X(0)=x$ obeys the normal distribution $N\left(0,\left(\left(\pi_{1} \sigma_{1}+\pi_{2} \sigma_{2}\right)^{2} / 2 \mid \pi_{1} \rho_{1}\right.\right.$ $\left.\left.+\pi_{2} \rho_{2} \mid\right)\right)$.

Now, if we choose $h_{0}\left(x_{1}\right)=e^{x_{1}}$, we can get that

$$
\begin{aligned}
E_{\mathrm{Q}}^{x_{1}}\left[h_{0}\left(X_{1}(T-t)\right)\right] & =E_{\mathrm{Q}}^{x_{1}}\left[\exp \left(X_{1}(T-t)\right)\right] \\
& =E_{\mathrm{Q}}\left[\exp \left\{x_{1} e^{\left(\pi_{1} \rho_{1}+\pi_{2} \rho_{2}\right)(T-t)}+\left(\pi_{1} \sigma_{1}+\pi_{2} \sigma_{2}\right) \int_{0}^{T-t} e^{\left(\pi_{1} \rho_{1}+\pi_{2} \rho_{2}\right)(T-t-s)} \mathrm{d} \widetilde{B}(s)\right\}\right] \\
& =\exp \left\{x_{1} e^{\left(\pi_{1} \rho_{1}+\pi_{2} \rho_{2}\right)(T-t)}+\frac{\left(\pi_{1} \sigma_{1}+\pi_{2} \sigma_{2}\right)^{2}}{4\left(\pi_{1} \rho_{1}+\pi_{2} \rho_{2}\right)}\left(e^{2\left[\left(\pi_{1} \rho_{1}+\pi_{2} \rho_{2}\right)(T-t)\right]}-1\right)\right\},
\end{aligned}
$$

for $\pi_{1} \rho_{1}+\pi_{2} \rho_{2} \neq 0$. So, Theorem 1 implies that

$$
\begin{aligned}
\phi_{0}(t, \omega)= & \frac{\mathrm{d}}{\mathrm{d} x_{1}} E_{Q}^{x_{1}}\left[h_{0}\left(X_{1}(T-t)\right)\right]_{x_{1}=X_{1}(t)}\left(\pi_{1} \sigma_{1}+\pi_{2} \sigma_{2}\right) \\
= & \left(\pi_{1} \sigma_{1}+\pi_{2} \sigma_{2}\right) e^{\left(\pi_{1} \rho_{1}+\pi_{2} \rho_{2}\right)(T-t)} \exp \left\{X_{1}(t) e^{\left(\pi_{1} \rho_{1}+\pi_{2} \rho_{2}\right)(T-t)}\right. \\
& \left.+\frac{\left(\pi_{1} \sigma_{1}+\pi_{2} \sigma_{2}\right)^{2}}{4\left(\pi_{1} \rho_{1}+\pi_{2} \rho_{2}\right)}\left(e^{2\left[\left(\pi_{1} \rho_{1}+\pi_{2} \rho_{2}\right)(T-t)\right]}-1\right)\right\},
\end{aligned}
$$

and from $\phi_{0}(t, \omega)=e^{\left(\pi_{1} \rho_{1}+\pi_{2} \rho_{2}\right) T} \phi(t, \omega)$, we get the expression of $\phi(t, \omega)$. Therefore, 


$$
\theta_{1}(t)= \begin{cases}e^{\left(\pi_{1} \rho_{1}+\pi_{2} \rho_{2}\right)(T-t)} \exp \left\{X_{1}(t) e^{\left(\pi_{1} \rho_{1}+\pi_{2} \rho_{2}\right)(T-t)}\right. & \\ \left.+\frac{\left(\pi_{1} \sigma_{1}+\pi_{2} \sigma_{2}\right)^{2}}{4\left(\pi_{1} \rho_{1}+\pi_{2} \rho_{2}\right)}\left(e^{2\left[\left(\pi_{1} \rho_{1}+\pi_{2} \rho_{2}\right)(T-t)\right]}-1\right)\right\}, & \text { if } \pi_{1} \rho_{1}+\pi_{2} \rho_{2} \neq 0, \\ \exp \left\{X_{1}(t)+\frac{\left(\pi_{1} \sigma_{1}+\pi_{2} \sigma_{2}\right)^{2}}{2}(T-t)\right\}, & \text { if } \pi_{1} \rho_{1}+\pi_{2} \rho_{2}=0 .\end{cases}
$$

Remark 5. We provide the results for the pricing and hedging of a European $T$-claim defined by the Ornstein-Uhlenbeck process with Markovian switching in example 2. If we let the generator of Markov chain $r(t)$ be $\Gamma=\left(\begin{array}{cc}-1 & 1 \\ 2 & -2\end{array}\right)$, then by solving linear equation (18), we have the result $\pi_{1}=2 / 3$ and $\pi_{2}=1 / 3$ that is the unique stationary (probability) distribution of $r(t)$. Taking the values of $\rho_{1}, \rho_{2}$, $\sigma_{1}$, and $\sigma_{2}$, we can explicitly get the expression of $\phi(t, \omega)$ and the portfolio $\theta(t)$ of $T$-claim $F(\omega)$.

\section{Concluding Remarks}

This paper mainly studied the Black-Scholes model with Markovian switching. The hedging of an attainable claim of a European option defined by this model is discussed. Under the assumption that Markov chain is irreducible, we obtained the explicit formula $\phi(t, \omega)$ and $p$ and $\theta(t)$ when $T$-claim $F(\omega)$ is given. An example of a market defined by an Ornstein-Uhlenbeck process is used to illustrate our theoretical results.

A business cycle is often divided into two or more different states, called "expansion" and "contraction" in financial economics. In this paper, we used a regime switching modulated by an irreducible Markov chain $r(t)$ to describe a business cycle. As described in the introduction, the adjustment of interest rates for the central bank will affect the operation of the economy and produce large economic fluctuations. For example, an interest rate increase will prompt investors to move their capitals towards the bank deposits. As a result, investments in stocks, options, and bonds will fall off. In contrast, decreased interest rates will cause capitals to flow into equities, options, and bonds. This will lead to the back-and-forth conversion of the option pricing between several models. The regime switching can be described by a Markov chain. For these reasons, it is necessary to consider the hedging and replication of an option pricing model under regime switching. Therefore, we carry out the pricing and hedging of an attainable claim for the European call options in a Black-Scholes model with Markovian switching.

The present paper is the first attempt, to our knowledge, to investigate the stochastic option pricing model with regime switching modulated by an irreducible Markov chain $r(t)$. We believe that parts of methods and results appearing in this paper are also available for other option pricing models, such as American option pricing model, Parisian option pricing models, and currency option pricing models. We leave this additional work for our future research.

\section{Data Availability}

Data are available upon reasonable request to the corresponding author.

\section{Conflicts of Interest}

The authors declare no conflicts of interest.

\section{Authors' Contributions}

All authors of this paper have equal contributions to this article, and all authors have read and approved the final manuscript.

\section{Acknowledgments}

The third author was supported by the Fundamental Research Funds for the Universities of Henan Province (NSFRF200321) and the Program of Young Scholar for Henan Polytechnic University (2020XQG-03).

\section{References}

[1] Q. Luo and X. Mao, "Stochastic population dynamics under regime switching," Journal of Mathematical Analysis and Applications, vol. 334, no. 1, pp. 69-84, 2007.

[2] Y. Takeuchi, N. H. Du, N. T. Hieu, and K. Sato, "Evolution of predator-prey systems described by a Lotka-Volterra equation under random environment," Journal of Mathematical Analysis and Applications, vol. 323, no. 2, pp. 938-957, 2006.

[3] X. Li, D. Jiang, and X. Mao, "Population dynamical behavior of Lotka-Volterra system under regime switching," Journal of Computational and Applied Mathematics, vol. 232, no. 2, pp. 427-448, 2009.

[4] X. Mao and C. Yuan, Stochastic Diffrential Equations with Markovian Switching, Imperial College Press, London, UK, 2006.

[5] R. S. Mamon and M. R. Rodrigo, "Explicit solutions to European options in a regime-switching economy," Operations Research Letters, vol. 33, no. 6, pp. 581-586, 2005.

[6] F. L. Yuen and H. Yang, "Option pricing with regime switching by trinomial tree method," Journal of Computational and Applied Mathematics, vol. 233, no. 8, pp. 1821-1833, 2010 . 
[7] J. Ma and T. Zhu, "Convergence rates of trinomial tree methods for option pricing under regime-switching models," Applied Mathematics Letters, vol. 39, pp. 13-18, 2015.

[8] B. Øksendal, Stochastic Differential Equations, SpringerVerlag, New York, NY, USA, 2003.

[9] I. Karatzas and D. Ocone, "A generalized Clark representation formula, with application to optimal portfolios," Stochastics and Stochastics Reports, vol. 34, pp. 187-220, 1991.

[10] Y. Hu, "Itô-Wiener chaos expansion with exact residual and correlation, variance inequalities," Journal of Theoretical Probability, vol. 10, no. 4, pp. 835-848, 1997.

[11] I. I. Gihman and A. V. Skorohod, Stochastic Differential Equations, Springer-Verlag, New York, NY, USA, 1974.

[12] M. Yor, Some Aspects of Brownian Motion, Part II, ETH Letctures in Math. Birkhauser, Basel, Switzerland, 1997. 